ARTICLE

Received 1 Jul 2013 | Accepted 26 Sep 2013 | Published 25 Oct 2013

DOI: $10.1038 /$ ncomms3684

OPEN

\title{
Localized cell stimulation by nitric oxide using a photoactive porous coordination polymer platform
}

Stéphane Diring ${ }^{1}$, Dan Ohtan Wang ${ }^{1}$, Chiwon Kim², Mio Kondoํ, Yong Chen¹, Susumu Kitagawa ${ }^{1,2}$, Ken-ichiro Kamei ${ }^{1} \&$ Shuhei Furukawa ${ }^{1}$

Functional cellular substrates for localized cell stimulation by small molecules provide an opportunity to control and monitor cell signalling networks chemically in time and space. However, despite improvements in the controlled delivery of bioactive compounds, the precise localization of gaseous biomolecules at the single-cell level remains challenging. Here we target nitric oxide, a crucial signalling molecule with site-specific and concentration-dependent activities, and we report a synthetic strategy for developing spatiotemporally controllable nitric oxide-releasing platforms based on photoactive porous coordination polymers. By organizing molecules with poor reactivity into polymer structures, we observe increased photoreactivity and adjustable release using light irradiation. We embed photoactive polymer crystals in a biocompatible matrix and achieve precisely controlled nitric oxide delivery at the cellular level via localized two-photon laser activation. The biological relevance of the exogenous nitric oxide produced by this strategy is evidenced by an intracellular change in calcium concentration, mediated by nitric oxide-responsive plasma membrane channel proteins.

\footnotetext{
${ }^{1}$ Institute for Integrated Cell-Material Sciences (WPI-iCeMS), Kyoto University, Yoshida, Sakyo-ku, Kyoto 606-8501, Japan. ${ }^{2}$ Department of Synthetic Chemistry and Biological Chemistry, Graduate School of Engineering, Kyoto University, Katsura, Nishikyo-ku, Kyoto 615-8510, Japan. Correspondence and requests for materials should be addressed to K.K. (email: kkamei@icems.kyoto-u.ac.jp) or to S.F. (email:shuhei.furukawa@icems.kyoto-u.ac.jp)
} 
C ontrolled cell stimulation by gaseous bioactive molecules is appealing for investigating cellular mechanisms and signalling networks, and for developing new therapeutic approaches ${ }^{1,2}$. The design of functional scaffolds or devices that can release molecules with precisely controlled timing, dosage and location remains challenging, especially for gaseous molecules, due to handling issues that arise from their high reactivity and physical state. Nitric oxide (NO) is one of the most investigated gasotransmitters, having a large role in numerous signalling events, including proliferation and vasodilatation ${ }^{3,4}$. Moreover, microenvironmental modulation by endogenous NO is believed to affect both physiological conditions, such as synaptic transmission, and also tissues and cancer stem cells ${ }^{5,6}$. Great effort has recently gone into the design of controllable NO-releasing scaffolds; however, the precise localization of NO delivery at the cellular level has not yet been demonstrated. We focus on the development of functional cellular substrates that provide ondemand NO release at multiple, well-defined locations.

Stable compounds that are able to produce NO through a photochemical reaction are a promising means of achieving temporal control over $\mathrm{NO}$ release, and a variety of $\mathrm{NO}$ photodonors have been recently developed ${ }^{7-9}$. Light provides a noninvasive trigger, which can be highly controlled in terms of intensity, wavelength or duration, without affecting any important physiological parameters. In addition, photons can be easily manipulated and focused to achieve precisely localized stimulation. To take full advantage of this spatial property, a concentration of photoresponsive molecules at defined locations is necessary for the consistent release of the target compound. Only a few examples of the functionalization of a nanoparticle surface $^{10}$ or the entrapment of photodonors into porous, nonfunctional scaffolds such as mesoporous silica ${ }^{11}$ have been reported.

Aside from other macromolecular scaffolds ${ }^{12}$, porous coordination polymers (PCPs) ${ }^{13-20}$ represent a distinct threedimensional (3D) framework, assembled from metal ions or clusters and functional organic ligands as building units. Designing appropriate NO photodonor ligands would advantageously render the framework itself photoactive, whereas the loading of NO donors into pores is no longer essential, and potentially toxic photoadducts remain chemically bound to the framework. The ligands are thereby concentrated in a restricted space that exhibits high light-harvesting and NOreservoir capacities. Moreover, the voids inherent in the framework can offer spatial segregation between the donors, thus preventing the aggregation-induced quenching of reactive excited species 21,22 .

In line with the strategy to apply PCPs in the biomedical field, relying on the accommodation and subsequent release of drug molecules from the pores ${ }^{19}$, PCP-based NO-releasing materials have been developed based on reversible chemical interactions between NO and accessible open metal sites in the framework $^{23,24}$. Recently, by taking advantage of the PCPs hybrid nature, functional ligands were post-synthetically modified into $\mathrm{N}$-diazeniumdiolate donors ${ }^{25,26}$. However, in each of these examples the coordination frameworks spontaneously release drug molecules or NO in physiological conditions and the delivery process cannot be initiated or interrupted at a specific time.

We propose a new approach for developing on-demand NO delivery platforms based on photoactive PCPs. The assembly of nitro-containing imidazolate ligands, which have low photoreactivity, into well-defined crystalline coordination frameworks leads to a drastic increase in photoreactivity, and the resulting materials can efficiently release NO, only on light irradiation. The applicability of these frameworks for localized cellular delivery is investigated by developing hybrid substrates in which micro- crystals of photoactive PCPs are embedded into a biocompatible and gas-permeable polymer matrix to allow cellular adhesion. Two-photon laser scanning confocal microscopy experiments allows for chemical modulation of the cellular microenvironment through the spatiotemporally controlled release of NO. The biological relevance of the delivered $\mathrm{NO}$ is demonstrated by a transient increase in the intracellular calcium ion concentration in locally stimulated cells following activation of the NO-sensitive membrane protein.

\section{Results}

Synthesis and characterization of NO frameworks. The development of photoactive PCPs ${ }^{27-30}$ is one of the powerful strategies towards on-demand control of porous properties. However, this approach has never been applied to photoinduced release of biologically relevant molecules. Inspired by $\mathrm{NO}$ photodonors based on nitroaromatic compounds, which undergo photoinduced nitro-to-nitrite rearrangement and subsequent bond cleavage to yield NO radicals ${ }^{31,32}$, we selected imidazole-based ligands, 2-nitroimidazole (2nIm) and 5-methyl-4-nitroimidazole $(\mathrm{mnIm})$, which are suitable for constructing robust and versatile zeolitic imidazolate frameworks (ZIFs) ${ }^{33-35}$. The solvothermal reaction of zinc nitrate with $2 \mathrm{nIm}$ or $\mathrm{mnIm}$ in dimethylformamide (DMF) yielded single crystals of $\left[\mathrm{Zn}(2 \mathrm{nIm})_{2}\right]_{n}(\mathrm{NOF}-1)$ or $\left[\mathrm{Zn}(\mathrm{mnIm})_{2}\right]_{n}(\mathrm{NOF}-2)$, respectively (NOF, NO framework). Alternatively, homogeneous microcrystalline powders of NOF-1 and NOF-2 were synthesized in a water/DMF mixture at room temperature in the presence of a sodium formate modulator, which induced faster nucleation by ligand deprotonation (Supplementary Fig. $\mathrm{S} 1)^{36}$. Single-crystal X-ray diffraction (XRD) measurements of both NOF-1 and NOF-2 (Supplementary Data 1 and 2) revealed that the imidazolate ligands bound the zinc ions with a tetrahedral coordination geometry, resulting in the formation of $3 \mathrm{D}$ porous coordination frameworks with sodalite (sod) topology. NOF- 1 crystallizes as cubic crystal system, $I-43 m$, which creates a perfectly truncated octahedral sodalite cage (Fig. 1a), whereas in NOF-2 the trigonal crystal system, $R-3 m$, exhibits an elongated sodalite cage along the $c$ axis (Fig. 1b). Twelve nitro functionalities are exposed in each sodalite cage without significant interaction with neighbouring atoms (Supplementary Fig. S2). The powder XRD measurements, together with the single-crystal XRD measurements, unambiguously confirmed that NOF-1 and NOF-2 are isostructural to the previously reported ZIF compounds, $\left[\mathrm{Co}(2 \mathrm{nIm})_{2}\right]_{n}$ (ref. 37) and $[\mathrm{Zn}(\mathrm{bIm})]_{n}$ (ref. 38), respectively (bIm, benzimidazole; Fig. 1c and Supplementary Tables S1 and S2).

To characterize the optical properties of the PCP frameworks, the solid-state ultraviolet-visible absorption spectra of NOF-1, NOF-2, $\left[\mathrm{Co}(2 \mathrm{nIm})_{2}\right]_{n}$ and the imidazole ligands, $2 \mathrm{nIm}$ and mnIm, were recorded (Fig. 2). Both ligands present broad and intense absorption bands in the $260-430 \mathrm{~nm}$ range, assigned to the $n-\pi^{*}$ and $\pi-\pi^{\star}$ transitions of the aromatic nitro functionalities. The similarity between the NOF-1 and $2 \mathrm{nIm}$ absorption spectra $\left(\lambda_{\max }=375 \mathrm{~nm}\right)$ indicates that the electronic environment of the ligand remains essentially unaffected on formation of the crystalline coordination framework; the $\mathrm{Zn}$ (II) ions do not become electronically involved in either the groundstate behaviour or the excited-state decay of their coordinating nitrogen-based ligands ${ }^{39}$. The situation is different for $\left[\mathrm{Co}(2 \mathrm{nIm})_{2}\right]_{n}$, the cobalt analogue of NOF-1; a slightly broadened and bathochromically shifted ultraviolet absorption band and new absorption bands appear in the visible region from 450 to $650 \mathrm{~nm}\left(\lambda_{\max }=550 \mathrm{~nm}\right)$, which can be assigned to the spin-allowed $d-d$ transitions $\left({ }^{4} \mathrm{~A}_{2}(\mathrm{~F}) \rightarrow{ }^{4} \mathrm{~T}_{2}(\mathrm{P})\right)$ of the $\mathrm{Co}(\mathrm{II})$ centres in a tetrahedral coordination environment ${ }^{40}$. 
a

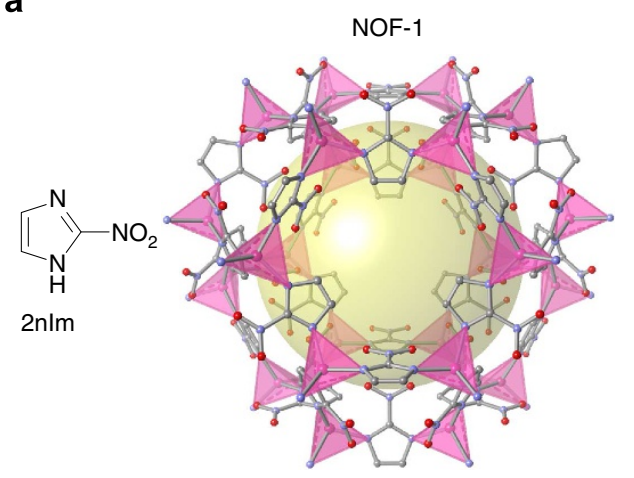

b

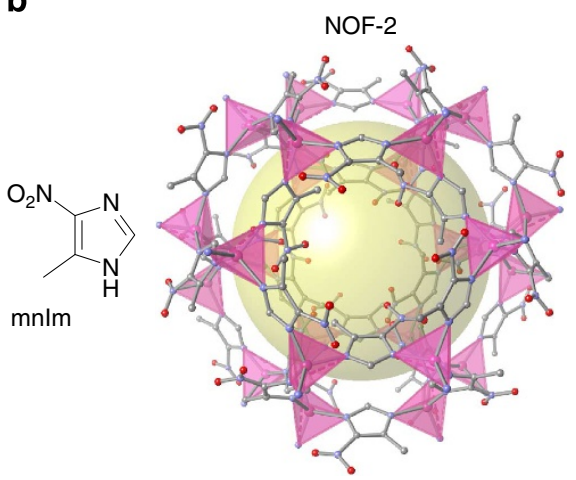

c

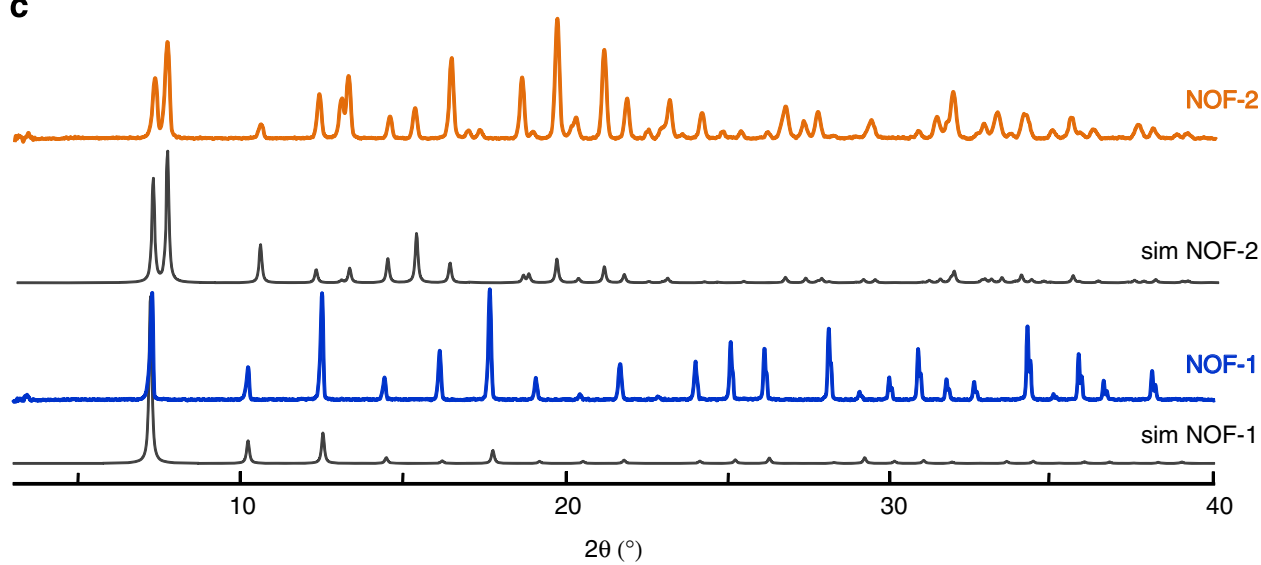

Figure 1 | Representation of the NO-releasing frameworks. $(\mathbf{a}, \mathbf{b})$ The nitro-containing imidazole ligands $2 \mathrm{nlm}$ and mnlm were independently reacted in the presence of zinc cations to form the porous sodalite zeolite imidazolate frameworks NOF-1 and NOF-2. (c) The powder XRD peaks of the NOF-1 and NOF-2 match the simulation patterns obtained from the single crystal diffraction data.

In turn, NOF-2 presents a redshifted absorption maximum $\left(\lambda_{\max }=450 \mathrm{~nm}\right)$ with respect to $\operatorname{mnIm}\left(\lambda_{\max }=410 \mathrm{~nm}\right)$, reflecting a more planar orientation of the nitro groups in the framework, thereby leading to an increase in electronic delocalization in the aromatic imidazole ring ${ }^{41}$.

Light-induced NO release. The NO-releasing properties of NOF-1 and NOF-2 under light irradiation (300-W Xenon lamp, $7.5 \mathrm{~mW} \mathrm{~cm}^{-2}$ ) were probed using the NO-selective ozone-chemiluminescence technique (Fig. 3a). First, the 2nIm and mnIm ligands presented low photoreactivity and produced only limited quantities of $\mathrm{NO}$ after $3 \mathrm{~h}$ of sustained illumination (release yield $=1.4 \%$ and $5.9 \%$ for $2 \mathrm{nIm}$ and $\mathrm{mnIm}$, respectively, based on the conversion of nitro groups to NO). However, when these ligands were organized into crystalline frameworks, their photoreactivity exhibited an increase greater than an order of magnitude under the same illumination conditions; NOF-1 and NOF-2 released 3.4 and $2.9 \mu \mathrm{mol} \mathrm{mg}^{-1}$ of $\mathrm{NO}$, corresponding to a conversion yield of $50 \%$ and $46 \%$, respectively.

Although the values are comparable, NOF-1 exhibits a moderately superior NO-releasing efficiency to that of NOF-2 (under a 300 - to $600-\mathrm{nm}$ light irradiation), which can be explained by the improved light-absorption capacity of NOF-1 (Fig. 2) due to the nitro substitution position on the imidazole ring; NOF-1 demonstrates significant absorption tailing in the range of $400-500 \mathrm{~nm}$.

The absorption spectra imply that the increase in the photoreactivity of the framework compounds over that of their respective ligands cannot be assigned to the perturbation of the electronic environment of the ligands upon $\mathrm{Zn}$ (II) coordination. As with the solid-state luminescence quenching effect observed in most fluorescent organic compounds, the inefficiency with which both mnIm and nIm release NO from their aggregated form on light irradiation can be explained by intermolecular interactions (favouring the formation of excimers) that provide efficient non-reactive deactivation pathways for the excited states ${ }^{42}$. In contrast, as observed from their crystal structures, the porous frameworks of NOF-1 and NOF-2 provide sufficient spatial segregation to prevent intermolecular ligand interactions and thus an excited-state deactivation ${ }^{21,22}$. The lack of NO production by the methylimidazolate-based reference material, $\left[\mathrm{Zn}(\mathrm{MeIm})_{2}\right]_{n}$ $(\text { ZIF-8 })^{36}$, supports the photochemical involvement of the nitro groups in the formation of NO (Supplementary Fig. S3). As confirmed by gas chromatography-mass spectrometry (GCMS) analysis, NO was the only nitrogen oxide species released after the irradiation of NOF-1 (Supplementary Fig. S4). Interestingly, the cobalt framework $\left[\mathrm{Co}(2 \mathrm{nIm})_{2}\right]_{n}$ failed to release significant quantities of NO (2\%) compared with NOF-1 (Fig. 2). As observed in the ultraviolet-visible spectrum, the ligand field and other low-energy states present on the cobalt centre can deactivate the excited states localized on the functional ligands and disfavour the photochemical deactivation pathway. These observations underline the importance of the choice in metal ion to the construction of efficient photoactive frameworks.

Furthermore, we obtained a two-dimensional non-porous framework, referred to as NOF-3, assembled from the $2 \mathrm{nIm}$ ligand and $\mathrm{Zn}(\mathrm{II})$ ions, $[\mathrm{Zn}(2 \mathrm{nIm})(\mathrm{HCOO})]_{n}$, by modifying the reaction conditions. Compared with NOF-1, the amount of $\mathrm{NO}$ released upon similar illumination was significantly reduced in the 

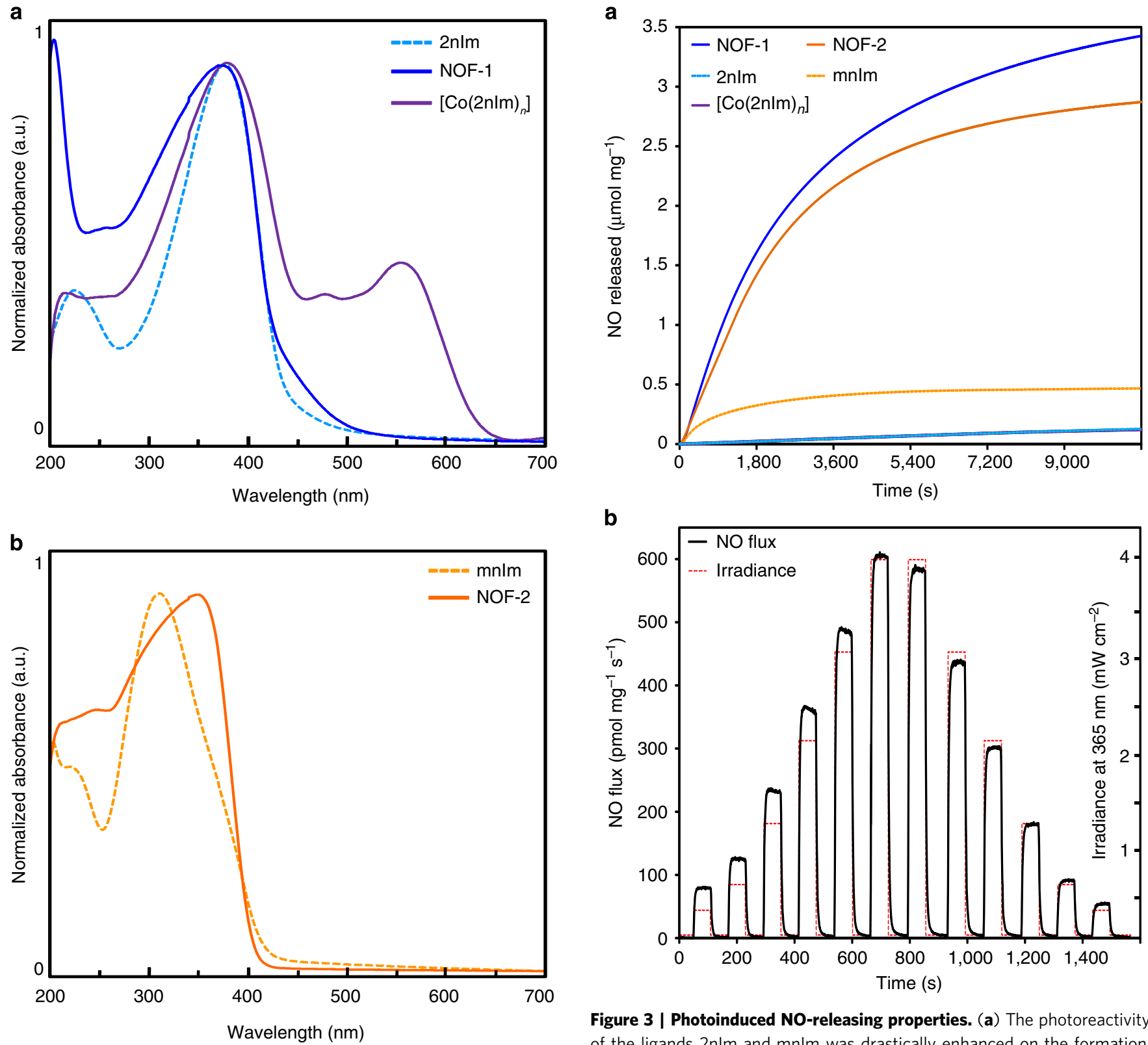

Figure 3 | Photoinduced NO-releasing properties. (a) The photoreactivity of the ligands $2 \mathrm{nlm}$ and $\mathrm{mnlm}$ was drastically enhanced on the formation of the PCPs NOF-1 and NOF-2. The $\left[\mathrm{Co}(2 \mathrm{nlm})_{2}\right]_{n}$ did not release a

Figure 2 | UV-Vis diffuse-reflectance spectra of NOF powders and their ligands. (a) The characteristic absorption band assigned to the transition located on the nitro functionality in $2 \mathrm{nlm}$ remains unaffected upon the coordination of $\mathrm{Zn}(\mathrm{II})$ in NOF-1. As anticipated, $\left[\mathrm{Co}(2 \mathrm{nlm})_{2}\right]_{n}$ gives rise to additional absorption bands in the visible region, which are characteristic of $\mathrm{Co}(\mathrm{II})$ in a tetrahedral coordination environment. (b) A bathochromic shift in the absorption band is observed in NOF-2, on the coordination of zinc, which suggests enhanced $\pi$-conjugation in the nitroaromatic ligand.

dense framework $\left(1.2 \mu \mathrm{mol} \mathrm{mg}^{-1}\right.$ after $3 \mathrm{~h}$; Supplementary Figs. S5-S9). This observation supports our design strategy through which efficient NO-releasing properties can be achieved by constructing suitable porous frameworks from NO photodonor building blocks.

In addition to high releasing efficiency, spatiotemporal control over the produced NO flux is important to NO delivery systems. We investigated the illumination of NOF-1 under variable light power (Fig. 3b and Supplementary Fig. S10). NO is released instantly on photoactivation and the NO flux can be easily tuned

by both the irradiation intensity and the amount of irradiated material. As anticipated, the production of NO is immediately interrupted when the irradiation ceases and its concentration quickly drops to trace levels. NOF powders did not present any significant decrease in crystallinity or in NO release after 8 months of storage under ambient conditions (Supplementary Fig. S11). In addition, the lack of significant NO release when heated up to $200^{\circ} \mathrm{C}$, confirmed the thermal stability of NOF powders and that NO is produced exclusively through a photochemical process (Supplementary Fig. S12). 
Spatiotemporally controlled release in cell media. To render our compounds biologically applicable, we prepared NOF-based substrates suitable for cell cultures and microscopic imaging (Fig. 4a); NOF-1 microcrystals were first spin-cast on a glassbottomed dish. Next, a biocompatible polydimethylsiloxane (PDMS) layer was spin-coated on top to embed the crystals completely in a polymer matrix with a reproducible thickness $(20 \pm 5 \mu \mathrm{m})$. Notably, the crystal distribution on the glass surface did not seem to be altered by the spin-coating process and the NOF crystals remained on the bottom part of the polymer layer (Fig. 4b and Supplementary Fig. S13). As confirmed by irradiation experiments, the thin PDMS layer allowed for the diffusion of released NO (Supplementary Fig. S14). Although moderately slowing the release kinetics, the total quantity of NO released remained comparable to that of the uncoated material. In addition, the fine tuning of the light intensity allowed a sustained and adjustable flux of NO for $2 \mathrm{~h}\left(250 \mathrm{pmol} \mathrm{mg}^{-1} \mathrm{~s}^{-1}\right.$ for $1 \mathrm{~h}$, then $350 \mathrm{pmol} \mathrm{mg}^{-1} \mathrm{~s}^{-1}$ for another hour by using increased light power; Supplementary Fig. S15).

To deliver NO in a localized cellular environment, we utilized the high spatiotemporal resolution of confocal laser scanning microscopy, which allowed for precise and localized photoactivation of the NOF-1/PDMS substrates and subsequent rapid observation of the cellular response. As the absorption of our material was essentially in the ultraviolet region, we used a nearinfrared two-photon laser to photoactivate NOF-1, thus greatly reducing the risk of photodamaging the cells with harmful ultraviolet irradiation while effectively inducing the NO release.

The sterilized NOF-1/PDMS were coated with Matrigel to facilitate cell adhesion and growth, and then human embryonic kidney 293 (HEK293) cells were cultured on this substrate (Supplementary Fig. S16). Compared with pristine PDMS substrates, the presence of NOF-1 in the polymer layer did not induce any notable cytotoxicity (Supplementary Fig. S17).

We predicted that the high spatial resolution of the two-photon confocal laser would allow for the selective activation of any crystals chosen to enable localized NO delivery to the surrounding cells. Before the activation experiments, DAF-FM DA (4amino-5-methylamino- $2^{\prime}, 7^{\prime}$-difluorofluorescein diacetate ${ }^{43}$, a cell-permeable NO fluorescent indicator, was introduced into the HEK293 cells. As shown in Fig. 4c,d, the rapid photoactivation of a selected region containing NOF-1 crystals quickly induced an increase in the fluorescence of the surrounding HEK293 cells.

Within seconds, the NO generated from NOF-1 was able to diffuse through the PDMS layer to the cellular membrane and further reacted with the NO indicator to form a fluorescent adduct. These results clearly suggest a high degree of spatiotemporal control over the NO delivery using a simple, reproducible and extremely fast two-photon photoactivation process. As control experiments, $\left[\mathrm{Zn}(\mathrm{MeIm})_{2}\right]_{n}$ microcrystals (lacking nitro functions) and an area of the PDMS substrate without any microcrystals were irradiated under identical photoactivation conditions. The irradiation did not induce any increase in the intracellular DAF-FM fluorescence in the surrounding cells (Supplementary Fig. S18).

Having demonstrated rapid NO delivery into cells with spatiotemporal precision, we aimed to probe the biological relevance of the NO released from photoactive PCP by observing an NO-activated cellular response. Calcium ions $\left(\mathrm{Ca}^{2+}\right)$ have a key role in regulating many physiological processes in mammalian cells, including neurotransmission, muscle contraction, proliferation, fertilization and apoptosis ${ }^{44}$. In this context, we targeted an NO-sensing transmembrane channel protein, transient receptor potential channel 5 (TRPC5), which undergoes a conformational change in the presence of $\mathrm{NO}$, allowing for
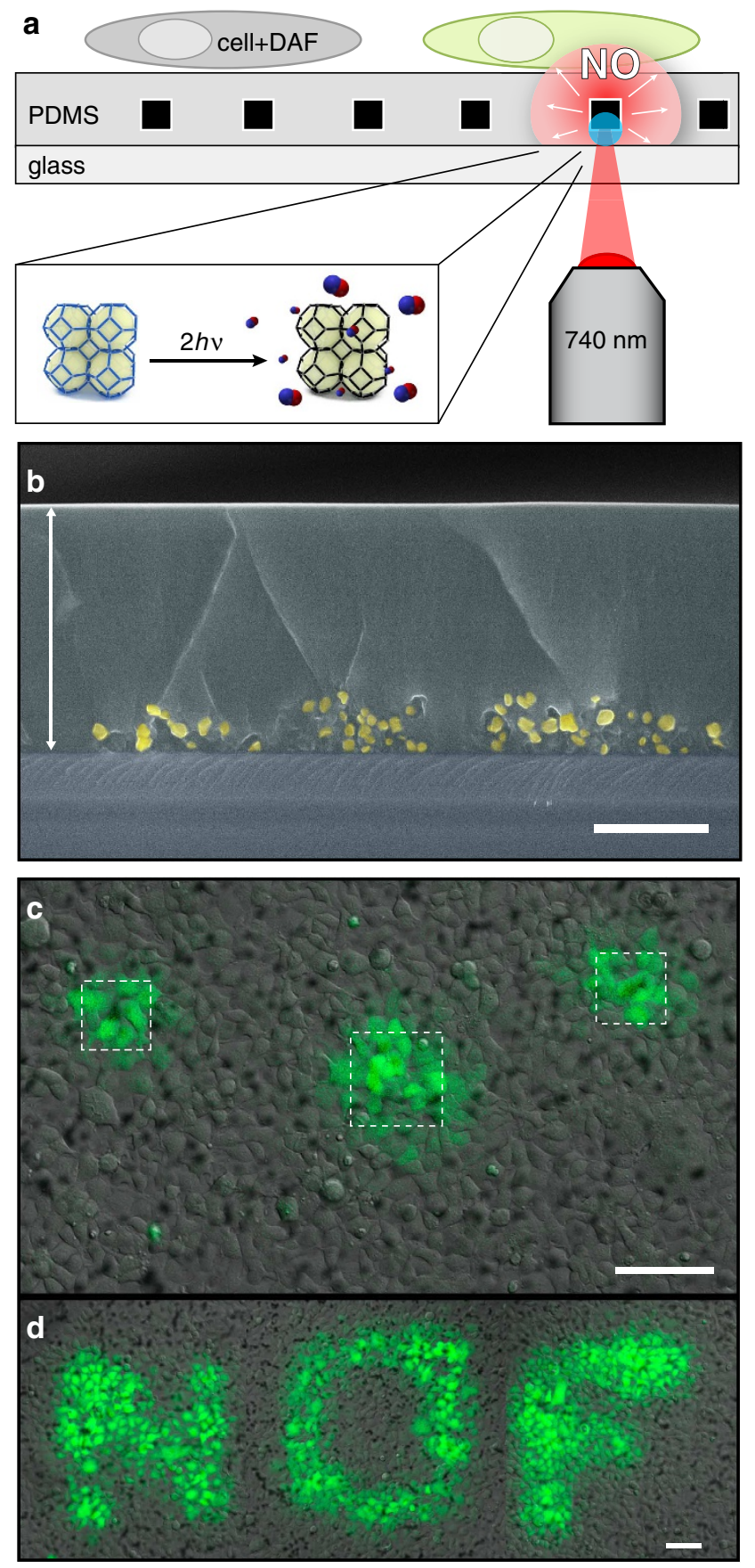

Figure 4 | Spatiotemporally controlled release of NO. (a) Schematic illustration of the localized cell-stimulation platform. The PDMS-embedded NOF-1 crystals are locally photoactivated by two-photon near-infrared laser irradiation. The generated NO diffuses through the PDMS layer and reacts with an intracellular NO fluorescent indicator, DAF-FM. (b) Cross-section scanning electron microscopy image of NOF-1/PDMS substrate. The white arrow highlights the homogeneous PDMS layer. NOF-1 crystals (yellow) are localized on the bottom part of the substrate, ensuring their isolation from the cellular medium (scale bar, $10 \mu \mathrm{m}$ ). (c) Confocal microscopy images of NOF-1-embedded substrates cultured with HEK293 cells introduced via DAF-FM. The selective photoactivation of the NOF-1 crystals (white squares) induced a fluorescent response in the surrounding cells, highlighting the localized NO delivery and uptake (scale bar,

$100 \mu \mathrm{m}$ ). (d) Further demonstration of spatiotemporal control by writing 'NOF' upon activation of the selected regions (scale bar, $100 \mu \mathrm{m}$ ). 
an influx of extracellular $\mathrm{Ca}^{2+}$ ion into the cells (Supplementary Fig. S19) ${ }^{45}$.

We investigated whether the photoactivated release of NO from NOF-1/PDMS substrates was able to change the intracellular calcium concentration, $\left[\mathrm{Ca}^{2+}\right]_{i}$, in HEK293 cells genetically modified to express the TRPC5 transmembrane channel (HEK293-TRPC5). After introducing a fluorescent $\mathrm{Ca}^{2+}$ indicator (Fluo-4) into the cells, we monitored the changes in $\left[\mathrm{Ca}^{2+}\right]_{i}$ in the native HEK293 and the modified HEK293-TRPC5 cells after the two-photon photoactivation of NOF-1. As depicted in Fig. 5, although the NO delivered to the control native HEK293 cells did not affect $\left[\mathrm{Ca}^{2+}\right]_{i}$, a rapid $\left[\mathrm{Ca}^{2+}\right]_{i}$ response in the surrounding HEK293-TRPC5 cells was observed upon photoactivation of comparable quantities of NOF-1 crystals (Supplementary Figs S20 and S21 and Supplementary Movies 1

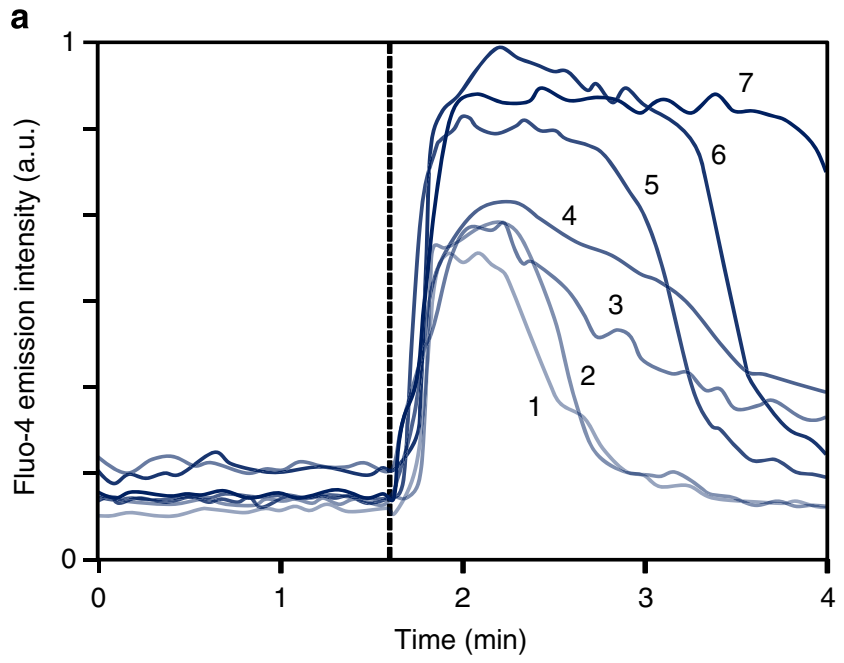

b

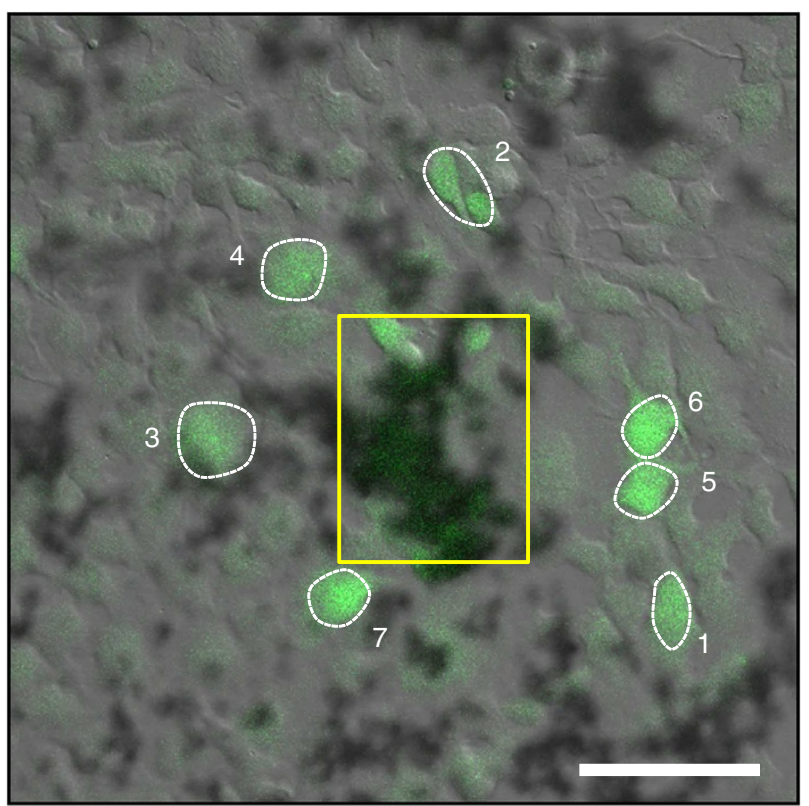

Figure 5 | Downstream signalling triggered by NO delivery. (a) Emission intensity profile of Fluo-4 in the HEK293-TRPC5 cells, accounting for the intracellular calcium ion concentration, $\left[\mathrm{Ca}^{2+}\right]_{i}$, before and after the photoactivation of NOF-1 (dashed line indicates initiation). The curves refer to the fluorescence of the circled cells in Fig. 5b. (b) Confocal microscopy image obtained after the irradiation of NOF-1 crystals (yellow rectangle) showing increased fluorescence of Fluo-4 in the HEK293-TRPC5 cells surrounding the photoactivation area (scale bar, $100 \mu \mathrm{m}$ ). and 2). These results indicate that NO released from the photoactivated PCP reacted with the TRPC5 channel on the cell membrane and triggered its opening. Note that the fluorescence intensity of Fluo-4 in the cells peaked after a few to several tens of seconds and, in most cases, decreased towards its initial level within a few minutes. This observation is in accordance with the transient nature of the NO delivery provided by the NOF-1 substrate with the rapid initiation and termination of its $\mathrm{NO}$ release. As previously observed in the NO-monitoring experiments conducted using DAF-FM, the irradiation of substrates incorporating $\left[\mathrm{Zn}(\mathrm{MeIm})_{2}\right]_{n}$ did not alter $\left[\mathrm{Ca}^{2+}\right]_{i}$ in the HEK293 or HEK293-TRPC5 cells (Supplementary Fig. S22).

\section{Discussion}

Light-induced NO release from macromolecular assemblies or solid-state materials has been achieved essentially by immobilizing NO photodonors on the surface of nanoparticles ${ }^{10}$ or accommodating them in host scaffolds such as mesoporous silica $^{11}$. Here we propose a different approach where the NO photodonors themselves are the building units, organized into $3 \mathrm{D}$ porous coordination structures. The spatial segregation of the functional components in porous frameworks, with electronically non-involved metal centres, allowed a drastic increase in the photo reactivity by preventing the quenching of the reactive excited states. This advantageous accumulation of photoactive donors in PCP architectures lead to efficient and tunable lightinduced NO release and places NOF materials $\left(3.4 \mu \mathrm{mol} \mathrm{mg}^{-1}\right.$ for NOF-1) above the most efficient, controllable solid-state NOdelivery scaffolds $\left(1.3 \mu \mathrm{mol} \mathrm{mg}{ }^{-1}\right.$ for nitrosothiol-modified silica xerogels $)^{12}$. Notably, their excellent stability under ambient conditions avoids the need of cautious handling or storage (as required with light-sensitive metal-nitrosyls or thermally unstable nitrosothiols). This work suggests a great potential of PCPs for controllable light-induced release of biologically relevant molecules.

To take advantage of the concentration of photodonor ligands in the PCP structure, we prepared a new NO cell-stimulation platform by immobilizing the NOF-1 microcrystals in a permeable and biocompatible polymer layer, and we confirmed that the irradiation of NOF-1/PDMS could generate relevant and sustained flux of gaseous NO, which easily diffused through the polymer layer. A high degree of spatiotemporal control over the NO delivery was achieved using harmless near-infrared twophoton laser activation and allowed for a precisely localized stimulation of cells surrounding the photoactivated crystals. We have further demonstrated the biological relevance of this exogenous NO delivery by observing the subsequent cellular response, that is, a transient increase in the intracellular calcium concentration, triggered by the conformational change in a NOsensitive transmembrane channel. Aside from the potential therapeutic applications, we believe this approach could provide valuable fundamental understanding of the physiological and pathophysiological roles of NO, particularly the functions of NO in brain circuitry in which the spatiotemporal generation of stimulating molecules in subcellular domains is desired.

\section{Methods}

Preparation of single crystals of NOF-1. The $2-\mathrm{nIm}(113 \mathrm{mg}, 1.0 \mathrm{mmol})$ and $\mathrm{Zn}\left(\mathrm{NO}_{3}\right)_{2} \cdot 6 \mathrm{H}_{2} \mathrm{O}(149 \mathrm{mg}, 0.5 \mathrm{mmol})$ were dissolved in DMF $(5 \mathrm{ml})$ in a sealed microwave vial. The solution was heated to $100{ }^{\circ} \mathrm{C}$ via microwave irradiation for $1 \mathrm{~h}$. Individual yellow crystals appeared on the glass. The yield could be increased by heating the mixture in a $100^{\circ} \mathrm{C}$ oven for $24 \mathrm{~h}$.

Preparation of single crystals of NOF-2. MnIm $(25 \mathrm{mg}, 0.20 \mathrm{mmol})$ and $\mathrm{Zn}\left(\mathrm{NO}_{3}\right)_{2} \cdot 6 \mathrm{H}_{2} \mathrm{O}(21 \mathrm{mg}, 0.07 \mathrm{mmol})$ were dissolved in DMF $(3 \mathrm{ml})$ in a glass tube The solution was heated in a $100^{\circ} \mathrm{C}$ electric oven. After $48 \mathrm{~h}$, light-yellow single crystals appeared on the glass. 
Preparation of single crystals of NOF-3. 2-Nitroimidazole ( $23 \mathrm{mg}, 0.20 \mathrm{mmol}$ ) and $\mathrm{Zn}\left(\mathrm{NO}_{3}\right)_{2} \cdot 6 \mathrm{H}_{2} \mathrm{O}(30 \mathrm{mg}, 0.10 \mathrm{mmol})$ were dissolved in a DMF- $\mathrm{H}_{2} \mathrm{O}$ mixture $(\mathrm{v} / \mathrm{v} 1 / 1,1 \mathrm{ml})$ in a glass tube and heated in an oil bath at $100^{\circ} \mathrm{C}$ for $36 \mathrm{~h}$ to afford pale yellow plate-like single crystals.

Preparation of microcrystalline NOF-1 and NOF-2 powders. $2-\mathrm{nIm}(113 \mathrm{mg}$, $1.0 \mathrm{mmol})$ or $\mathrm{mnIm}(127 \mathrm{mg}, 1.0 \mathrm{mmol})$ and sodium formate $(136 \mathrm{mg}, 2.0 \mathrm{mmol})$ were dissolved in a $\mathrm{DMF} / \mathrm{H}_{2} \mathrm{O}(\mathrm{v} / \mathrm{v}, 10 / 1)$ mixture $(5 \mathrm{ml})$. A solution of $\mathrm{Zn}\left(\mathrm{NO}_{3}\right)_{2} \cdot 6 \mathrm{H}_{2} \mathrm{O}(149 \mathrm{mg}, 0.5 \mathrm{mmol})$ in $\mathrm{H}_{2} \mathrm{O}(5 \mathrm{ml})$ was later added, and the reaction mixture was stirred vigorously at room temperature. The reaction mixture became cloudy within a few minutes after adding the zinc solution. After $18 \mathrm{~h}$, the precipitate was centrifuged and washed with water $(2 \times 15 \mathrm{ml})$ and DMF

$(3 \times 15 \mathrm{ml})$ to yield NOF-1 $(68 \mathrm{mg}, 27 \%)$ as a pale yellow powder or NOF-2 $(35 \mathrm{mg}$, $13 \%$ ) as a white powder. A solvent-exchange procedure was performed by immersing the powders successively in fresh methanol $(4 \times 15 \mathrm{ml}$ over $48 \mathrm{~h})$. The compounds were then heated under reduced pressure $\left(80^{\circ} \mathrm{C}, 10 \mathrm{mbar}\right)$ for $18 \mathrm{~h}$. Anal. calculated for NOF-1, $\mathrm{C}_{6} \mathrm{H}_{4} \mathrm{~N}_{6} \mathrm{O}_{4} \mathrm{Zn}=\mathrm{Zn}(2 \mathrm{nIm})_{2}$ : C, 24.89; H, 1.39; N, 29.03; found: C, 24.77; H, 1.61; N, 28.08. Anal. calculated for NOF-2,

$\mathrm{C}_{11} \mathrm{H}_{15} \mathrm{~N}_{7} \mathrm{O}_{5} \mathrm{Zn}=\mathrm{Zn}(\mathrm{mnIm})_{2}$. (DMF): C, 33.82; H, 3.87; N, 25.10; found: C, 33.27; $\mathrm{H}, 3.57 ; \mathrm{N}, 25.20$

Preparation of microcrystalline NOF-3 powder. 2-Nitroimidazole $(113 \mathrm{mg}$, $1.00 \mathrm{mmol})$ and $\mathrm{Zn}\left(\mathrm{NO}_{3}\right)_{2} \cdot 6 \mathrm{H}_{2} \mathrm{O}(297 \mathrm{mg}, 1.00 \mathrm{mmol})$ were dissolved in DMF $(10 \mathrm{ml})$ by microwave irradiation at $100^{\circ} \mathrm{C}$ for $6 \mathrm{~h}$. The resulting product was washed with DMF $(3 \times)$ to afford a pale yellow powder $(200 \mathrm{mg}, 90 \%)$. Anal. calculated for $\mathrm{C}_{4} \mathrm{H}_{3} \mathrm{~N}_{3} \mathrm{O}_{4} \mathrm{Zn}=\mathrm{Zn}(2 \mathrm{nIm})\left(\mathrm{HCO}_{2}\right): \mathrm{C}, 21.59 ; \mathrm{H}, 1.36 ; \mathrm{N}, 18.89$; found: $\mathrm{C}, 21.35 ; \mathrm{H}, 1.37 ; \mathrm{N}, 18.67$.

Preparation of [Co(2nIm)2] (ZIF-65). 2-Nitroimidazole (90 mg, $0.80 \mathrm{mmol}$ ) and $\mathrm{Co}\left(\mathrm{NO}_{3}\right)_{2} \cdot 6 \mathrm{H}_{2} \mathrm{O}(120 \mathrm{mg}, 0.40 \mathrm{mmol})$ were dissolved in DMF $(4 \mathrm{ml})$ in a glass vial. The solution was heated in an electric oven for $6 \mathrm{~h}$ at $120^{\circ} \mathrm{C}$ and then cooled down to room temperature within $12 \mathrm{~h}$. Block crystal of $[\mathrm{Co}(2 \mathrm{nIm}) 2]_{n}$ formed on the glass vial. The mother liquor was quickly filtered while hot. After few minutes, a microcrystalline powder of ZIF-65 precipitated at room temperature. The purple powder was centrifuged and washed with $\operatorname{DMF}(3 \times)$. Anal. calculated for $\mathrm{C}_{9} \mathrm{H}_{13} \mathrm{CoN}_{7} \mathrm{O}_{6}=\mathrm{Co}(2 \mathrm{nIm})_{2} \cdot(\mathrm{DMF})\left(\mathrm{H}_{2} \mathrm{O}\right): \mathrm{C}, 28.89 ; \mathrm{H}, 3.50 ; \mathrm{N}, 26.20$; found: C, 28.81; H, 3.46; N, 25.58 .

Crystal structure determination. Single crystals of NOF-1, NOF-2 or NOF-3 were placed on a MicroLoops with Paraton-N (Hampton Research) and mounted on a Rigaku AFC10 diffractometer with Rigaku Saturn Kappa CCD system equipped with a MicroMax-007 HF/VariMax rotating-anode X-ray generator with confocal monochromatized $\operatorname{MoK} \alpha(\gamma=0.71069 \AA)$ radiation. The crystal structures were solved by a direct method and refined by full-matrix least-squares refinement using the SHELXL-97 (Supplementary Tables S1-S3 and Supplementary Data 1-3). All non-hydrogen atoms were refined anisotropically with hydrogen atoms generated as spheres riding the coordinates of their parent atoms. Solvent molecules were removed by SQUEEZE procedure.

Powder X-ray diffraction. Measurements were performed with a Bruker D8 operating with $\mathrm{Cu} \mathrm{K} \alpha$ radiation $(\lambda=1.54 \AA$ ) with a $40-\mathrm{kV}$ beam voltage and 40-mA current.

Field-emission scanning electron microscopy. Observations were performed with a JEOL Model JSM-7001F4 scanning electron microscopy system operating at $15.0 \mathrm{kV}$. The samples were deposited on carbon tape and coated with osmium before measurement.

Gas chromatography mass spectroscopy. Analyses were performed with a Shimadzu GCMS-QP5050A, using a ZB-1 (polymethylsyloxane) column $\left(60 \mathrm{~m} \times 0.32 \mathrm{~mm}\right.$ and $5.0 \mu \mathrm{m}$ thickness). The column temperature was $40^{\circ} \mathrm{C}$ and the temperature of injection was $200{ }^{\circ} \mathrm{C}$ with helium gas carrier (pressure $80 \mathrm{kPa}$; flow rate, $2 \mathrm{ml} \mathrm{min}^{-1}$ ). Mass spectrometer was operated at $20{ }^{\circ} \mathrm{C}$ with an ionization voltage of $70 \mathrm{eV}$ and ionization current of $60 \mathrm{~mA}$.

Light-induced NO release. Methanol suspensions of NOF microcrystals $\left(\sim 2 \mathrm{mg} \mathrm{ml}^{-1}\right)$ were repeatedly spin-cast $(2,000$ r.p.m. $)$ on a $22 \times 22$-mm cover slip and activated under reduced pressure for $3 \mathrm{~h}\left(10 \mathrm{mbar}, 85^{\circ} \mathrm{C}\right)$. The weight of the deposited material was accurately determined using a precision scale (typical weights, $80-120 \mu \mathrm{g}$ ). The samples were placed in a custom-made cell and irradiated with a 300-W Xenon lamp (Asahi Spectra Max-303 equipped with a 300- to 600-nm ultraviolet-visible module and $\times 1.0$ collimator lens). The incident light power at $365 \mathrm{~nm}$ was measured using an Ushio UIT-201 intensity meter. The NO release measurements were performed using a Sievers NOA 280i chemiluminescence NO analyser. The instrument was calibrated by passing air through a zero filter (Sievers,
$<1 \mathrm{ppb} \mathrm{NO}$ ) and $640 \mathrm{ppb} \mathrm{NO}$ gas. The nitrogen carrier gas flow rate was set to $200 \mathrm{ml} \mathrm{min}^{-1}$ with a cell pressure of 8.5 Torr and an oxygen pressure of $6.1 \mathrm{psig}$.

Substrate preparation. A methanolic suspension of NOF-1 was repeatedly spincast $(2,000$ r.p.m.) onto a glass-bottomed culture dish. The crystals were embedded in a PDMS layer by spin-coating a pre-polymer/curing agent mixture (w/w, 10/1, Sylgard 184) at 3,000 r.p.m. for $1 \mathrm{~min}$ to allow the formation of a reproducible 15to $20-\mu \mathrm{m}$-thick polymer layer (determined via laser scanning confocal microscopy and scanning electron microscopy). The substrate was heated at $80^{\circ} \mathrm{C}$ under reduced pressure for an additional $18 \mathrm{~h}$.

Cell culture. The HEK293 and HEK293-TRPC5 cells were cultured in medium consisting of DMEM supplemented with $10 \%(\mathrm{v} / \mathrm{v})$ fetal bovine serum and $1 \%(\mathrm{v} / \mathrm{v})$ penicillin/streptomycin in a humidified incubator $\left(37^{\circ} \mathrm{C}, 5 \% \mathrm{CO}_{2}\right)$. The medium was changed every $2-3$ days. The cells were passaged with trypsin-EDTA solutions. For the HEK293-TRPC5 cells, $100 \mu \mathrm{g} \mathrm{ml}^{-1} \mathrm{G} 418$ was added to the culture medium for their selection.

Cell culture on an NOF-1/PDMS substrate. Before cell culture, a NOF-1/PDMS substrate was sterilized using $70 \%$ ethanol and coated with Matrigel (BD Bioscience, USA; certified for human embryonic stem cell culture) that had been diluted with DMEM/Ham F12 medium to facilitate cell adhesion and growth at $4{ }^{\circ} \mathrm{C}$ overnight. The substrate was then rinsed with cell culture medium twice to remove any excess Matrigel. The trypsinized HEK293 or HEK293-TRPC5 cells $\left(1 \times 10^{5}\right.$ cells $)$ were introduced onto a substrate and cultured until the experiments were performed. For the HEK293-TRPC5 cells, a culture medium with $100 \mu \mathrm{g} \mathrm{ml}^{-1}$ G418 was used, but replaced with fresh culture medium without G418 1 day before the experiments.

Fluorescence imaging. A Fluo- 4 (molecular probe; $1 \mu \mathrm{M}$ ) or DAF-FM (molecular probe; $5 \mu \mathrm{M}$ ) was introduced into the cells in culture medium $30 \mathrm{~min}$ before the imaging experiments. After the incubation period, the cells were quickly rinsed once with PBS and imaged in Tyrode's solution $(129 \mathrm{mM} \mathrm{NaCl}, 5 \mathrm{mM} \mathrm{KCl}, 2 \mathrm{mM}$ $\mathrm{CaCl}_{2}, 1 \mathrm{mM} \mathrm{MgCl}_{2}, 30 \mathrm{mM}$ glucose, $25 \mathrm{mM}$ HEPES and $0.01 \mathrm{mM}$ glycine).

Laser scanning confocal microscopy experiments. All fluorescent images used for cell imaging were acquired on an Axio Observer Z1 mounted with a live-cell imaging chamber, a motorized XY-stage, a Plan-Apochromat $20 \times / 0.80$ objective (LSM780, Zeiss) and a Chameleon VISION II (680-1,080 nm) two-photon laser (Coherent Inc.). The imaging parameters for the Fluo-4 and DAF-FM (excitation at $488 \mathrm{~nm}$, emission at $493 / 545 \mathrm{~nm}$ ) were determined based on information provided by the manufacturer.

Apoptosis assay. Cell apoptosis were detected with Annexin V apoptosis detection kit APC (eBioscience). Briefly, cells were washed twice with PBS and collected with $0.25 \%$ trypsin in PBS (Invitrogen). Cells were suspended in cell culture medium and placed in 15-ml tubes. After centrifugation at 1,000 r.p.m. for $5 \mathrm{~min}$, the supernatant was removed. Cells were washed with PBS and binding buffer (eBioscience), and then counted. For staining, cells were diluted to $5 \times$ 106 cells $\mathrm{ml}^{-1}$ in binding buffer. Annexin V labelled with allophycocyanin were added and incubated at room temperature for $15 \mathrm{~min}$. Cells were centrifuged at 1,000 r.p.m. for $5 \mathrm{~min}$ and then washed with binding buffer. Cells were centrifuged and resuspended in binding buffer to which propidium iodide was added. Labelled cells were analysed with a FACS Canto II (BD Biosciences).

\section{References}

1. Szabó, C. Hydrogen sulphide and its therapeutic potential. Nat. Rev. Drug Discov. 6, 917-935 (2007).

2. Motterlini, R. \& Otterbein, L. E. The therapeutic potential of carbon monoxide. Nat. Rev. Drug Discov. 9, 728-743 (2010).

3. Murad, F. Discovery of some of the biological effects of nitric oxide and its role in cell signaling (Nobel Lecture). Angew. Chem. Int. Ed. 38, 1856-1868 (1999)

4. Fukumura, D., Kashiwagi, S. \& Jain, R. K. The role of nitric oxide in tumour progression. Nat. Rev. Cancer 6, 521-534 (2006).

5. Vincent, S. R. Nitric oxide neurons and neurotransmission. Prog. Neurobiol. 90, 246-255 (2010).

6. Eyler, C. E. et al. Glioma stem cell proliferation and tumor growth are promoted by nitric oxide synthase-2. Cell 146, 53-66 (2011).

7. Sortino, S. Light-controlled nitric oxide delivering molecular assemblies. Chem Soc. Rev. 39, 2903-2913 (2010)

8. Fry, N. L. \& Mascharak, P. K. Photoactive ruthenium nitrosyls as NO donors: how to sensitize them toward visible light. Acc. Chem. Res. 44, 289-298 (2011)

9. Riccio, D. A. et al. Nitric oxide-releasing S-nitrosothiol-modified xerogels. Biomaterials 30, 4494-4502 (2009). 
10. Caruso, E. B., Petralia, S., Conoci, S., Giuffrida, S. \& Sortino, S. Photodelivery of nitric oxide from water-soluble platinum nanoparticles. J. Am. Chem. Soc. 129, 480-481 (2007).

11. Heilman, St B. J., John, J., Oliver, S. R. J. \& Mascharak, P. K. Light-triggered eradication of acinetobacter baumannii by means of NO delivery from a porous material with an entrapped metal nitrosyl. J. Am. Chem. Soc. 134, 11573-11582 (2012).

12. Riccio, D. A. \& Schoenfisch, M. H. Nitric oxide release: part I. Macromolecular scaffolds. Chem. Soc. Rev. 41, 3731-3741 (2012).

13. Yaghi, O. M. et al. Reticular synthesis and the design of new materials. Nature 423, 705-714 (2003).

14. Kitagawa, S., Kitaura, R. \& Noro, S. Functional porous coordination polymers. Angew. Chem. Int. Ed. 43, 2334-2375 (2004).

15. Hurd, J. A. et al. Anhydrous proton conduction at $150{ }^{\circ} \mathrm{C}$ in a crystalline metal-organic framework. Nat. Chem. 1, 705-710 (2009).

16. Robone, J. et al. An adaptable peptide-based porous material. Science 329, 1053-1057 (2010).

17. Mohideed, M. I. H. et al. Protecting group and switchable pore-discriminating adsorption properties of a hydrophilic-hydrophobic metal-organic framework. Nat. Chem. 3, 304-310 (2011).

18. Bloch, E. D. et al. Hydrocarbon separations in a metal-organic framework with open iron(III) coordination sites. Science 335, 1606-1610 (2012).

19. Horcajada, P. et al. Porous metal-organic-framework nanoscale carriers as a potential platform for drug delivery and imaging. Nat. Mater. 9, 172-178 (2009).

20. Della Rocca, J., Liu, D. \& Lin, W. Nanoscale metal-organic frameworks for biomedical imaging and drug delivery. Acc. Chem. Res. 44, 957-968 (2011).

21. Takashima, Y. et al. Molecular decoding using luminescence from an entangled porous framework. Nat. Commun. 2, 168 (2011).

22. Lee, C. Y. et al. Light-harvesting metal-organic frameworks (MOFs): efficient strut-to-strut energy transfer in bodipy and porphyrin-based MOFs. J. Am. Chem. Soc. 133, 15858-15861 (2011).

23. McKinlay, A. C. et al. Exceptional behavior over the whole adsorption-storagedelivery cycle for NO in porous metal organic frameworks. J. Am. Chem. Soc. 130, 10440-10444 (2008)

24. Hinks, N. J., McKinlay, A. C., Xiao, B., Wheatley, P. S. \& Morris, R. E. Metal organic frameworks as NO delivery materials for biological applications. Micropor. Mesopor. Mater. 129, 330-334 (2010).

25. Nguyen, J. G., Tanabe, K. K. \& Cohen, S. M. Postsynthetic diazeniumdiolate formation and NO release from MOFs. CrystEngComm 12, 2335-2338 (2010).

26. Ingleson, M. J., Heck, R., Gould, J. A. \& Rosseinsky, M. J. Nitric oxide chemisorption in a postsynthetically modified metal - organic framework. Inorg. Chem. 48, 9986-9988 (2009).

27. Sato, H., Matsuda, M., Sugimoto, K., Takata, M. \& Kitagawa, S. Photoactivation of a nanoporous crystal for on-demand guest trapping and conversion. Nat. Mater. 9, 661-666 (2010)

28. Blake, A. et al. Photoreactivity examined through incorporation in metal-organic frameworks. Nat. Chem. 2, 688-694 (2010).

29. Tanabe, K. K., Allen, C. A. \& Cohen, S. M. Photochemical activation of a metal-organic framework to reveal functionality. Angew. Chem. Int. Ed. 49, 9730-9733 (2010)

30. Deshpande, R. K. et al. Photolabile protecting groups in metal-organic frameworks: preventing interpenetration and masking functional groups. Chem. Commun. 48, 1574-1576 (2012).

31. Galloway, D. B., Bartz, J. A., Huey, L. G. \& Fleming Crim, F. Pathways and kinetic energy disposal in the photodissociation of nitrobenzene. J. Chem. Phys. 98, 2107-2114 (1993).

32. Fukuhara, K., Kurihara, M. \& Miyata, N. Photochemical generation of nitric oxide from 6-nitrobenzo[a]pyrene. J. Am. Chem. Soc. 123, 8662-8666 (2001).

33. Phan, A. et al. Synthesis, structure, and carbon dioxide capture properties of zeolitic imidazolate frameworks. Acc. Chem. Res. 43, 58-67 (2010).

34. Tan, J. C., Bennett, T. D. \& Cheetham, A. K. Chemical structure, network topology, and porosity effects on the mechanical properties of zeolitic imidazolate frameworks. Proc. Natl Acad. Sci. USA 107, 9938-9943 (2010).

35. Huang, X. C., Lin, Y. Y., Zhang, J. P. \& Chen, X. M. Ligand-directed strategy for zeolite-type metal-organic frameworks: $\operatorname{zinc}(\mathrm{II})$ imidazolates with unusual zeolitic topologies. Angew. Chem. Int. Ed. 45, 1557-1559 (2006).
36. Cravillon, J. et al. Controlling zeolitic imidazolate framework nano- and microcrystal formation: insight into crystal growth by time-resolved in situ static light scattering. Chem. Mater. 23, 2130-2141 (2011).

37. Banerjee, R. et al. High-throughput synthesis of zeolitic imidazolate frameworks and application to CO2 capture. Science 319, 939-943 (2008).

38. Park, K. S. et al. Exceptional chemical and thermal stability of zeolitic imidazolate frameworks. Proc. Natl Acad. Sci. USA 103, 10186-10191 (2006).

39. Muro, M. L., Diring, S., Wang, X., Ziessel, R. \& Castellano, F. N. Photophysics of the platinum(II) terpyridyl terpyridylacetylide platform and the influence of FeII and ZnII coordination. Inorg. Chem. 47, 6796-6803 (2008).

40. Tonigold, M. et al. Pyrazolate-based cobalt(II)-containing metal-organic frameworks in heterogeneous catalytic oxidation reactions: elucidating the role of entatic states for biomimetic oxidation processes. Chem. Eur. J. 17, 8671-8695 (2011).

41. Sortino, S. et al. The photochemistry of flutamide and its inclusion complex with $\beta$-Cyclodextrin. Dramatic effect of the microenvironment on the nature and on the efficiency of the photodegradation pathways. Photochem. Photobiol 73, 6-13 (2001).

42. Jenekhe, S. A. \& Osaheni, J. A. Excimers and exciplexes of conjugated polymers Science 265, 765-768 (1994).

43. Kojima, H. et al. Fluorescent indicators for imaging nitric oxide production. Angew. Chem. Int. Ed. 38, 3209-3212 (1999).

44. Berridge, M. J., Lipp, P. \& Bootman, M. D. The versatility and universality of calcium signalling. Nat. Rev. Mol. Cell Biol. 1, 11-21 (2000).

45. Yoshida, T. et al. Nitric oxide activates TRP channels by cysteine S-nitrosylation. Nat. Chem. Biol. 2, 596-607 (2006).

\section{Acknowledgements}

We thank Professor Y. Mori and Dr. N. Takahashi for supplying HEK293-TRPC5 cells, K. Shiomi for assistance with GSMS experiments and CeMI for assistance with confocal microscopy and electron microscopy. We thank M. Nakajima, M. Nakashima,

M. Yoshioka and Y. Koyama for their experimental assistance. C.K. is grateful to JSPS Research Fellowships for Young Scientists. This work was supported by ERATO 'Kitagawa Integrated Pores Project' of the Japan Science and Technology Agency and Grants-in-Aid for Scientific Research from MEXT (No. 24750159 for Wakate B (S.D.), No. 24108720 for Priority Area 'Coordination Programming' (S.F) and No. 23681028 for Wakate A (K.K.)). iCeMS is supported by the World Premier International Research Initiative (WPI), MEXT, Japan.

\section{Author contributions}

S.D., K.K. and S.F. conceived and designed the experiments. S.D. and C.K. performed all synthetic and characterization experiments. S.D., C.K. and M.K. performed crystallographic analysis. S.D., D.O.W. and K.K. performed confocal microscope imaging experiments. Y.C. and S.K. supervised the project. S.D., K.K. and S.F. analysed the data and wrote the manuscript. All authors discussed the results and commented on the manuscript.

\section{Additional information}

Accession codes: The X-ray crystallographic coordinates and structure factors for NOF-1, NOF-2 and NOF-3 have been deposited at the Cambridge Crystallographic Data Centre (CCDC) under accession codes 917446 to 917448 , respectively. These data can be obtained free of charge from the Cambridge Crystallographic Data Centre via http:// www.ccdc.cam.ac.uk/data_request/cif.

Supplementary Information accompanies this paper at http://www.nature.com/ naturecommunications

Competing financial interests: The authors declare no competing financial interests.

Reprints and permission information is available online at http://npg.nature.com/ reprintsandpermissions/

How to cite this article: Diring, S. et al. Localized cell stimulation by nitric oxide using a photoactive porous coordination polymer platform. Nat. Commun. 4:2684 doi: $10.1038 /$ ncomms3684 (2013).

This work is licensed under a Creative Commons AttributionNonCommercial-NoDerivs 3.0 Unported License. To view a copy of this license, visit http://creativecommons.org/licenses/by-nc-nd/3.0/ 